\title{
Deformation quantization of compact Kähler manifolds by Berezin-Toeplitz quantization
}

\author{
Martin Schlichenmaier \\ Department of Mathematics and Computer Science, University of Mannheim, D7, 27 \\ D-68131 Mannheim, Germany \\ e-mail: schlichenmaier@math.uni-mannheim.de
}

\begin{abstract}
For arbitrary compact quantizable Kähler manifolds it is shown how a natural formal deformation quantization (star-product) can be obtained via Berezin-Toeplitz operators. Results on their semi-classical behaviour (their asymptotic expansion) due to Bordemann, Meinrenken, and Schlichenmaier are used in an essential manner. It is shown that the star-product is null on constants and fulfills parity. A trace is constructed and the relation to deformation quantization by geometric quantization is given.
\end{abstract}

Keywords: quantization, Kähler manifolds, star-products, semi-classical limit, Toeplitz structure

Mathematics Subject Classifications (2000): 58F06, 58F05, 53C55, 32C17, $81 \mathrm{~S} 10$

Dedicated to the memory of Moshé Flato

\section{Introduction}

By Bayen, Flato, Fronsdal, Lichnerowicz, and Sternheimer in 1977 the important concept of quantization given by deforming the algebra of functions in "direction" of the Poisson bracket was introduced [1]. Clearly the intuitive concept of $\hbar$-depending "deformation" of classical mechanics into quantum mechanics was around earlier (e.g. Weyl quantization). But in their work a mathematically very precise meaning was given to it.

Since this time the existence of a deformation quantization for every symplectic manifold was established in different ways. Some of the persons involved were De Wilde and Lecomte [11], Fedosov [15], and Omori, Maeda, and Yoshioka [27]. Quite recently this was extended to every Poisson manifold by Kontsevich [21]. Classification results are also available $[4,13,26,14,36]$.

Even if there is now a very general existence theorem it is still of importance to study deformation quantizations for such manifolds which carry additional geometric structures. From the whole set of deformation quantization one is looking for one which keeps the additional structure. In this spirit the article deals with the deformation quantization of compact quantizable Kähler manifolds. It was shown 1993 by Bordemann, Meinrenken, and Schlichenmaier [6] that for compact quantizable Kähler manifolds the Berezin-Toeplitz quantization has the correct semi-classical behaviour (see Theorem 2.3 below). Shortly after [6] was submitted we had also the result that by the techniques developed there it was possible to 
construct a deformation quantization [12]. Details were written up in German [32] and the result (with few steps of the proof) appeared in [31, 33]. The complete proof was not published in English.

Compact Kähler manifolds appear as phase spaces of constrained systems and as reduced phase spaces under a group action. More recently, they play a rather prominent role in Chern-Simons theory, topological and 2-dimensional conformal field theory. Here typically, the phase spaces to be quantized are moduli spaces of certain geometric objects. As examples the compactified moduli spaces of stable holomorphic vector bundles (maybe with additional structures) on a Riemann surface show up. The quantum Hilbert spaces appearing in this context are the Verlinde spaces.

Encouraged by the recent interest in deformation quantization evolving in these fields I found it worthwhile to publish the above mentioned results also in English and add some pieces to it. By the construction of the deformation quantization direct relations to the Berezin-Toeplitz quantization, the geometric quantization (via Tuynman's relation), and asymptotic operator representations are given. Hence what is presented here is more than just another existence proof.

In the proof the theory of generalized Toeplitz operators developed by Boutet de Monvel and Guillemin [9, 16] is used in an essential manner. In the article [17] which appeared one year later than [6] it was also explained by Guillemin himself in which way the existence of a deformation quantization follows from the general theory of Toeplitz operators. More precisely, he showed that the subalgebra of operators commuting with the $S^{1}$ action (with respect to the sphere bundle $Q$ defined in Section 3) modulo Toeplitz operators of degree $-\infty$ defines via the symbol map a deformation quantization. This follows from results on the principal and subprincipal symbols proved in [9]. Note that essentially the same idea was employed in [6], in the answer to [12], in [31, 32], and will be presented here. Hence, the deformation quantizations obtained will coincide. One might even say that suitable reinterpreted nowadays from the point of view of deformation quantization their existence was implicitly already contained (at least to a certain extend) in the Boutet de Monvel - Guillemin theory of Toeplitz operators.

Only for certain special examples of compact Kähler manifolds direct constructions have been known earlier; see results by Berezin [3], Moreno and OrtegaNavarro [23, 24], and Cahen, Gutt, and Rawnsley [10]. Recently, for all Kähler manifolds (including the noncompact ones) the existence of a deformation quantization with "separation of variables" was shown by Karabegov [18]. Separation of variables says essentially that the deformation quantization "respects" the complex structure. A classification of all such deformation quantizations for a fixed Kähler manifold was also given by Karabegov. Note that his existence proof is on the level of the formal deformation quantization. It does not yield Hilbert spaces and quantum operators like in our approach (which in contrast is restricted to the case of quantizable compact Kähler manifolds). Independently, a similar existence theorem was proven by Bordemann and Waldmann [7] along Fedosov's 
original approach. Yet another construction was given recently by Reshetikhin and Takhtajan [28].

Finally, let me stress the fact, that the very essential basics of this work go back to joint work with Martin Bordemann and Eckhard Meinrenken. Details have been added by me later on.

The article is organized as follows. In Section 2 the geometric set-up is given and the main result of this article, the theorem on the construction of the deformation quantization (Theorem 2.2) is formulated. The approximation results from [6] are recalled. In Section 3 the necessary details about the Toeplitz structure introduced by Boutet de Monvel and Guillemin are given. They are employed in Section 4 for the construction of the deformation quantization (the star-product), i.e., the proof of Theorem 2.2. In the concluding Section 5 additional properties of the star-product are discussed. It is shown that we have $1 \star g=g \star 1=g$, i.e., that the star-product is "null on constants" and that it fulfills the parity condition. A trace is constructed. By a result of Tuynman for compact Kähler manifolds the geometric quantization can be expressed in terms of the Berezin-Toeplitz quantization. Using our theorem we see that the geometric quantization yields also a star-product. This star-product is equivalent to the constructed one. The BerezinToeplitz star-product will be a local star-product given by bidifferential operators. It will have the property of "separation of variables". This will be shown in [19].

\section{The set-up and the main result}

Let $(M, \omega)$ be a compact (complex) Kähler manifold of complex dimension $n$. It should be considered as phase space manifold $M$ with symplectic form given by the Kähler form $\omega$. Denote by $C^{\infty}(M)$ the algebra of (arbitrary often) differentiable functions. Using the Kähler form one assigns to every $f \in C^{\infty}(M)$ its Hamiltonian vector field $X_{f}$ and to every pair of functions $f$ and $g$ the Poisson bracket:

$$
\omega\left(X_{f}, \cdot\right)=d f(\cdot), \quad\{f, g\}:=\omega\left(X_{f}, X_{g}\right) .
$$

With the Poisson bracket $C^{\infty}(M)$ becomes a Poisson algebra.

Assume $(M, \omega)$ to be quantizable. This says that there exists an associated quantum line bundle $(L, h, \nabla)$ with holomorphic line bundle $L$, Hermitian metric $h$ on $L$ and connection $\nabla$ compatible with the metric $h$ and the complex structure such that the curvature of the line bundle and the Kähler form $\omega$ of the manifold are related as

$$
\operatorname{curv}_{L, \nabla}(X, Y):=\nabla_{X} \nabla_{Y}-\nabla_{Y} \nabla_{X}-\nabla_{[X, Y]}=-\mathrm{i} \omega(X, Y) .
$$

Equation (2) is called the quantization condition. If the metric is represented as a function $\hat{h}$ with respect to local complex coordinates and a local holomorphic frame of the bundle the quantization condition reads as $\mathrm{i} \bar{\partial} \partial \log \hat{h}=\omega$.

The quantization condition implies that $L$ is a positive line bundle. By the Kodaira embedding theorem $L$ is ample, which says that a certain tensor power 
$L^{m_{0}}$ of $L$ is very ample, i.e., the global holomorphic sections of $L^{m_{0}}$ can be used to embed the phase space manifold $M$ into projective space. Note that the embedding is an embedding as complex manifolds not as Kähler manifolds. The embedding dimension is given by the Hirzebruch-Riemann-Roch formula. Hence, quantizable compact Kähler manifolds are as complex manifolds projective algebraic manifolds. The converse is also true, see [31,2]. In the following we will assume $L$ to be very ample. If $L$ is not very ample we choose $m_{0} \in \mathbb{N}$ such that the bundle $L^{m_{0}}$ is very ample and take this bundle as quantum line bundle and $m_{0} \omega$ as Kähler form for $M$. The underlying complex manifold structure is not changed. Please note that for the examples of moduli spaces mentioned in the introduction there is often a natural ample or very ample quantum line bundle.

We take the Liouville measure $\Omega=\frac{1}{n !} \omega^{n}$ as volume form on $M$. On the space of $C^{\infty}$-sections $\Gamma_{\infty}(M, L)$ we have the scalar product and norm

$$
\langle\varphi, \psi\rangle:=\int_{M} h(\varphi, \psi) \Omega, \quad\|\varphi\|:=\sqrt{\langle\varphi, \varphi\rangle} .
$$

Let $\mathrm{L}^{2}(M, L)$ be the $\mathrm{L}^{2}$-completion of the space of $C^{\infty}$-sections of the bundle $L$ and $\Gamma_{h o l}(M, L)$ be its (due to compactness of $M$ ) finite-dimensional closed subspace of holomorphic sections. Let $\Pi: \mathrm{L}^{2}(M, L) \rightarrow \Gamma_{h o l}(M, L)$ be the projection.

Definition 2.1 For $f \in C^{\infty}(M)$ the Toeplitz operator $T_{f}$ is defined to be

$$
T_{f}:=\Pi(f \cdot): \quad \Gamma_{h o l}(M, L) \rightarrow \Gamma_{h o l}(M, L) .
$$

In words: One takes a holomorphic section $s$ and multiplies it with the differentiable function $f$. The resulting section $f \cdot s$ will only be differentiable. To obtain a holomorphic section one has to project it back on the subspace of holomorphic sections.

The linear map $T: C^{\infty}(M) \rightarrow \operatorname{End}\left(\Gamma_{\text {hol }}(M, L)\right), f \rightarrow T_{f}$, is the Berezin-Toeplitz quantization map. Because in general $T_{f} T_{g}=\Pi(f \cdot) \Pi(g \cdot) \Pi \neq \Pi(f g \cdot) \Pi=T_{f g}$, it is neither a Lie algebra homomorphism nor an associative algebra homomorphism. From the point of view of Berezin's approach [3] the operator $T_{f}$ has as a contravariant symbol $f$ (see also [34] for relations to Berezin's covariant symbols).

This defines a map from the commutative algebra of functions to a noncommutative finite-dimensional (matrix) algebra. The finite-dimensionality is due to compactness of $M$. A lot of classical information will get lost. To recover this information one should consider not just the bundle $(L, \nabla, h)$ alone but all its tensor powers $\left(L^{m}, \nabla^{(m)}, h^{(m)}\right)$ and apply the above constructions for every $m$. Note that if $\hat{h}$ corresponds to the metric $h$ w.r.t. a holomorphic frame $s$ of the bundle $L$ then $\hat{h}^{m}$ corresponds to the metric $h^{(m)}$ w.r.t. to the frame $s^{\otimes m}$ for the bundle $L^{m}$. In this way one obtains a family of matrix algebras and a family of maps

$$
T^{(m)}: C^{\infty}(M) \rightarrow \operatorname{End}\left(\Gamma_{h o l}\left(M, L^{m}\right)\right), \quad f \rightarrow T_{f}^{(m)} .
$$


This infinite family should in some sense "approximate" the algebra $C^{\infty}(M)$. (See [5] and the discussion on strict quantization below.) for a definition of such an approximation.) Indeed this family has the correct semi-classical behaviour as is expressed in Theorem 2.3 below.

It also allows to construct a deformation quantization. A deformation quantization is given by a star-product. I will use both terms interchangeable. To fix the notation and the factors of i let me recall the definition of a star-product. Let $\mathscr{A}=C^{\infty}(M)[[v]]$ be the algebra of formal power series in the variable $v$ over the algebra $C^{\infty}(M)$. A product $\star$ on $\mathscr{A}$ is called a (formal) star-product if it is an associative $\mathbb{C}[[\boldsymbol{V}]]$-linear product such that

1. $\mathscr{A} / v \mathscr{A} \cong C^{\infty}(M)$, i.e., $f \star g \bmod v=f \cdot g$,

2. $\frac{1}{v}(f \star g-g \star f) \bmod v=-\mathrm{i}\{f, g\}$,

where $f, g \in C^{\infty}(M)$. We can also write

$$
f \star g=\sum_{j=0}^{\infty} C_{j}(f, g) v^{j},
$$

with $C_{j}(f, g) \in C^{\infty}(M)$. The $C_{j}$ should be $\mathbb{C}$-bilinear in $f$ and $g$. Conditions 1 and 2 can be reformulated as

$$
C_{0}(f, g)=f \cdot g, \quad \text { and } \quad C_{1}(f, g)-C_{1}(g, f)=-\mathrm{i}\{f, g\} .
$$

The aim of this article is to show the following

Theorem 2.2 There exists a unique (formal) star-product on $C^{\infty}(M)$

$$
f * g:=\sum_{j=0}^{\infty} v^{j} C_{j}(f, g), \quad C_{j}(f, g) \in C^{\infty}(M),
$$

in such a way that for $f, g \in C^{\infty}(M)$ and for every $N \in \mathbb{N}$ we have with suitable constants $K_{N}(f, g)$ for all $m$

$$
\left\|T_{f}^{(m)} T_{g}^{(m)}-\sum_{0 \leq j<N}\left(\frac{1}{m}\right)^{j} T_{C_{j}(f, g)}^{(m)}\right\|=K_{N}(f, g)\left(\frac{1}{m}\right)^{N} .
$$

This theorem has been proven immediately after [6] was finished. It has been announced in [31, 33] and the proof was written up in German in [32]. In Section 4 I will supply the proof.

Instead of writing (9) we will sometimes use the more intuitive notation

$$
T_{f}^{(m)} \cdot T_{g}^{(m)} \sim \sum_{j=0}^{\infty}\left(\frac{1}{m}\right)^{j} T_{C_{j}(f, g)}^{(m)} \quad(m \rightarrow \infty) .
$$

The asymptotics should always be understood in the above precise sense. 
In the proof the results expressed in the following theorem are needed. Denote by $\|f\|_{\infty}$ the sup-norm of $f$ on $M$ and by $\left\|T_{f}^{(m)}\right\|=\sup _{s \in \Gamma_{h o l}\left(M, L^{m}\right), s \neq 0} \frac{\left\|T_{f}^{(m)} s\right\|}{\|s\|}$ the operator norm on $\Gamma_{h o l}\left(M, L^{m}\right)$.

Theorem 2.3. (Bordemann, Meinrenken, Schlichenmaier)

(a) For every $f \in C^{\infty}(M)$ there exists $C>0$ such that

$$
\|f\|_{\infty}-\frac{C}{m} \leq\left\|T_{f}^{(m)}\right\| \leq\|f\|_{\infty} .
$$

In particular, $\lim _{m \rightarrow \infty}\left\|T_{f}^{(m)}\right\|=\|f\|_{\infty}$.

(b) For every $f, g \in C^{\infty}(M)$

$$
\left\|m \mathrm{i}\left[T_{f}^{(m)}, T_{g}^{(m)}\right]-T_{\{f, g\}}^{(m)}\right\|=O\left(\frac{1}{m}\right) \quad \text { as } \quad m \rightarrow \infty .
$$

(c) For every $f, g \in C^{\infty}(M)$

$$
\left\|T_{f}^{(m)} T_{g}^{(m)}-T_{f \cdot g}^{(m)}\right\|=O\left(\frac{1}{m}\right) \text { as } m \rightarrow \infty .
$$

These results are contained in Theorem 4.1, 4.2, resp. in Section 5 in [6]. Note that part (c) also follows from (9) for $N=1$ and generalizes trivially to finitely many functions.

Our result does not prove a strict deformation quantization in the sense of Rieffel [29]. But it is a strict quantization (see for the definition [22, 30]). Let $I:=\left\{\frac{1}{m} \mid m \in \mathbb{N}\right\} \cup\{0\}$ be the topological space with topology coming from the real line. It has 0 as accumulation point. To every $\hbar \in I, \hbar \neq 0$, i.e $\hbar=1 / m$, one assigns the algebra $A_{1 / m}:=\operatorname{End}\left(\Gamma_{h o l}\left(M, L^{m}\right)\right)$ with $\|\cdot\|_{1 / m}$ the operator norm. To 0 one assigns the algebra $A_{0}:=C^{\infty}(M)$ with norm $\|\cdot\|_{0}=|\cdot|_{\infty}$. The map $\hbar \rightarrow T_{f}^{(1 / \hbar)}$, with $T_{f}^{(\infty)}:=f$ defines by Theorem 2.3 a continuous field of $C^{*}$-algebras on the family $\left(A_{\hbar}\right)_{\hbar \in I}$. From (45) follows that $T$ respects conjugation. By (12) the additional condition for a strict quantization is also fulfilled. Due to the compactness of $M$ the maps $T_{f}^{(1 / \hbar)}$ for $\hbar \neq 0$ are never injective. Hence the strict quantization is not faithful at a fixed level $\hbar$, only in the limit $\hbar \rightarrow \infty$.

In [5] and [6] the notion of $L_{\alpha}$, resp. $g l(N)$, resp. $s u(N)$ quasi-limit was used for this concept. It was conjectured in [5] that for every compact Kähler manifold the Poisson algebra of function is a $g l(N)$ quasi-limit. This was proven in [6]. This result is of special interest in the theory of membranes.

There is another geometric concept of quantization, the geometric quantization introduced by Kostant and Souriau. But for compact Kähler manifolds due to Tuynman [35] (see also [5] for a coordinate independent proof) they have the same semi-classical behaviour

$$
Q_{f}^{(m)}=\mathrm{i} \cdot T_{f-\frac{1}{2 m} \Delta f}^{(m)} .
$$


Here $Q_{f}^{(m)}$ is the well-known operator of geometric quantization (with respect to the quantum line bundle $L^{m}$ ) corresponding to the prequantum operator $P_{f}^{(m)}=-\nabla_{X_{f}^{(m)}}^{(m)}+\mathrm{i} f \cdot i d$ and Kähler polarization. Kähler polarization means $Q_{f}^{(m)}=\Pi^{(m)} P_{f}^{(m)} \Pi^{(m)}$ with the projectors

$$
\Pi^{(m)}: \mathrm{L}^{2}\left(M, L^{m}\right) \rightarrow \Gamma_{h o l}\left(M, L^{m}\right) .
$$

In (14), $\Delta$ is the Laplacian with respect to the Kähler metric given by $\omega$. In Section $5 \mathrm{I}$ will show that this allows to define a deformation quantization via the operators of geometric quantization. It will be equivalent to the Berezin-Toeplitz deformation quantization.

\section{Toeplitz structure}

In [6] the set-up for the proof of the approximation results was given. Here I use the same setting. Let me recall for further reference the main definitions. A more detailed exposition can be found in [32]. Take $(U, k):=\left(L^{*}, h^{-1}\right)$ the dual of the quantum line bundle, $Q$ the unit circle bundle inside $U$ (with respect to the metric $k$ ) and $\tau: Q \rightarrow M$ the projection. Note that for the projective space with quantum line bundle the hyperplane section bundle $H$, the bundle $U$ is just the tautological bundle. Its fibre over the point $z \in \mathbb{P}^{N}(\mathbb{C})$ consists of the line in $\mathbb{C}^{N+1}$ which is represented by $z$. In particular, for the projective space the total space of $U$ with the zero section removed can be identified with $\mathbb{C}^{N+1} \backslash\{0\}$. The same picture remains true for the via the very ample quantum line bundle in projective space embedded manifold $M$. The quantum line bundle will be the pull-back of $H$ (i.e., its restriction to the embedded manifold) and its dual is the pull-back of the tautological bundle.

In the following we use $E \backslash 0$ to denote the total space of the vector bundle $E$ with the image of the zero section removed. Starting from the function $\hat{k}(\lambda):=$ $k(\lambda, \lambda)$ on $U$ we define $\tilde{a}:=\frac{1}{2 \mathrm{i}}(\partial-\bar{\partial}) \log \hat{k}$ on $U \backslash 0$ (with respect to the complex structure on $U$ ) and denote by $\alpha$ its restriction to $Q$. Now $d \alpha=\tau^{*} \omega$ (with $d=d_{Q}$ ) and $\mu=\frac{1}{2 \pi} \tau^{*} \Omega \wedge \alpha$ is a volume form on $Q$. With respect to this form we take the $\mathrm{L}^{2}$-completion $\mathrm{L}^{2}(Q, \mu)$ of the space of functions on $Q$. The generalized Hardy space $\mathscr{H}$ is the closure of the functions in $\mathrm{L}^{2}(Q, \mu)$ which can be extended to holomorphic functions on the whole disc bundle. The generalized Szegö projector is the projection

$$
\Pi: \mathrm{L}^{2}(Q, \mu) \rightarrow \mathscr{H} .
$$

By the natural circle action $Q$ is a $S^{1}$-bundle and the tensor powers of $U$ can be viewed as associated bundles. The space $\mathscr{H}$ is preserved by this action. It can be decomposed into eigenspaces $\mathscr{H}=\prod_{m=0}^{\infty} \mathscr{H}^{(m)}$ where $c \in S^{1}$ acts on $\mathscr{H}^{(m)}$ as multiplication by $c^{m}$. Sections of $L^{m}=U^{-m}$ can be identified with functions $\phi$ on $Q$ which satisfy the equivariance condition $\phi(c \lambda)=c^{m} \phi(\lambda)$. It turns out that this 
identification is an isometry. Recall that $\mathrm{L}^{2}\left(M, L^{m}\right)$ has a scalar product given in an corresponding way to (3). Restricted to the holomorphic objects we obtain an isometry

$$
\Gamma_{h o l}\left(M, L^{m}\right) \cong \mathscr{H}^{(m)} .
$$

There is the notion of Toeplitz structure $(\Pi, \Sigma)$ as developed by Boutet de Monvel and Guillemin in $[9,16]$. What is needed from there are only the following facts. $\Pi$ is the Szegö projector (16). The second object is the submanifold

$$
\Sigma=\{t \alpha(\lambda) \mid \lambda \in Q, t>0\} \subset T^{*} Q \backslash 0
$$

of the tangent bundle of $Q$ defined with the help of the 1-form $\alpha$. They showed that it is a symplectic submanifold. A (generalized) Toeplitz operator of order $k$ is an operator $A: \mathscr{H} \rightarrow \mathscr{H}$ of the form $A=\Pi \cdot R \cdot \Pi$ where $R$ is a pseudodifferential

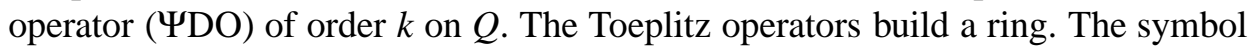
of $A$ is the restriction of the principal symbol of $R$ (which lives on $T^{*} Q$ ) to $\Sigma$. Note that $R$ is not fixed by $A$, but Guillemin and Boutet de Monvel showed that the symbols are well-defined and that they obey the same rules as the symbols of YDOs. In particular we have the following relations

$$
\sigma\left(A_{1} A_{2}\right)=\sigma\left(A_{1}\right) \sigma\left(A_{2}\right), \quad \sigma\left(\left[A_{1}, A_{2}\right]\right)=\mathrm{i}\left\{\sigma\left(A_{1}\right), \sigma\left(A_{2}\right)\right\}_{\Sigma} .
$$

In our context only two Toeplitz operators appear:

(1) The generator of the circle action gives the operator $D_{\varphi}=\frac{1}{\mathrm{i}} \frac{\partial}{\partial \varphi}$. It is an operator of order 1 with symbol $t$. It operates on $\mathscr{H}^{(m)}$ as multiplication by $m$.

(2) For $f \in C^{\infty}(M)$ let $M_{f}$ be the operator on $\mathrm{L}^{2}(Q, \mu)$ corresponding to multiplication with $\tau^{*} f$. We set ${ }^{1} T_{f}=\Pi \cdot M_{f} \cdot \Pi: \mathscr{H} \rightarrow \mathscr{H}$. Because $M_{f}$ is constant along the fibres of $\tau, T_{f}$ commutes with the circle action. Hence $T_{f}=\prod_{m=0}^{\infty} T_{f}^{(m)}$, where $T_{f}^{(m)}$ denotes the restriction of $T_{f}$ to $\mathscr{H}^{(m)}$. After the identification of $\mathscr{H}^{(m)}$ with $\Gamma_{h o l}\left(M, L^{m}\right)$ we see that these $T_{f}^{(m)}$ are exactly the Toeplitz operators $T_{f}^{(m)}$ introduced in Section 2. In this sense $T_{f}$ is called the global Toeplitz operator and the $T_{f}^{(m)}$ the local Toeplitz operators. $T_{f}$ is an operator of order 0 . Let us denote by $\tau_{\Sigma}: \Sigma \subseteq T^{*} Q \rightarrow Q \rightarrow M$ the composition then we obtain for the symbol $\sigma\left(T_{f}\right)=\tau_{\Sigma}^{*}(f)$.

\section{Proof of Theorem 2.2}

Let the notation be as in the last section. In particular, let $T_{f}$ be the Toeplitz operator, $D_{\varphi}$ the operator of rotation, and $T_{f}^{(m)}$, resp. $(m \cdot)$ their projections on the eigenspaces $\mathscr{H}^{(m)} \cong \Gamma_{h o l}\left(M, L^{m}\right)$.

\footnotetext{
1 There should be no confusion with the operator $T_{f}=T_{f}^{(1)}$ introduced above.
} 


\subsection{The definition of the $C_{j}(f, g) \in C^{\infty}(M)$}

The construction is done inductively in such a way that

$$
A_{N}=D_{\varphi}^{N} T_{f} T_{g}-\sum_{j=0}^{N-1} D_{\varphi}^{N-j} T_{C_{j}(f, g)}
$$

is always a Toeplitz operator of order zero. The operator $A_{N}$ is $S^{1}$-invariant, i.e., $D_{\varphi} \cdot A_{N}=A_{N} \cdot D_{\varphi}$. Because it is of order zero his symbol is a function on $Q$. By the $S^{1}$-invariance the symbol is even given by (the pull-back of) a function on $M$. Take this function to be the next element $C_{N}(f, g)$ in the star-product. By construction the operator $A_{N}-T_{C_{N}(f, g)}$ is of order -1 and $A_{N+1}=D_{\varphi}\left(A_{N}-T_{C_{N}(f, g)}\right)$ is of order 0 and hence exactly of the form given in (20). The induction starts with

$$
\begin{gathered}
A_{0}=T_{f} T_{g}, \quad \text { and } \\
\sigma\left(A_{0}\right)=\sigma\left(T_{f}\right) \sigma\left(T_{g}\right)=\tau_{\Sigma}^{*}(f) \cdot \tau_{\Sigma}^{*}(g)=\tau_{\Sigma}^{*}(f \cdot g) .
\end{gathered}
$$

Hence, $C_{0}(f, g)=f \cdot g$ as required.

It remains to show statement (9) about the asymptotics. As an operator of order zero on a compact manifold $A_{N}$ is bounded. Hence the same is true for all its restrictions $A_{N}^{(m)}$ to $\mathscr{H}^{(m)}$. If we calculate them we obtain

$$
\left\|m^{N} T_{f}^{(m)} T_{g}^{(m)}-\sum_{j=0}^{N-1} m^{N-j} T_{C_{j}(f, g)}^{(m)}\right\|=\left\|A_{N}^{(m)}\right\| \leq\left\|A_{N}\right\| .
$$

After dividing by $m^{N}$ Equation (9) follows. Bilinearity is clear.

\subsection{The Poisson structure}

The relation $C_{0}(f, g)=f \cdot g$ was proven above. To show the 2 . formula in (7) we write explicitly (23) for $N=2$ and the pair of functions $(f, g)$ :

$$
\left\|m^{2} T_{f}^{(m)} T_{g}^{(m)}-m^{2} T_{f \cdot g}^{(m)}-m T_{C_{1}(f, g)}^{(m)}\right\| \leq K .
$$

A corresponding expression is obtained for the pair $(g, f)$. If we subtract both operators inside of the norm we obtain (with the triangle inequality and suitable $K^{\prime}$ )

$$
\left\|m^{2}\left(T_{f}^{(m)} T_{g}^{(m)}-T_{g}^{(m)} T_{f}^{(m)}\right)-m\left(T_{C_{1}(f, g)}^{(m)}-T_{C_{1}(g, f)}^{(m)}\right)\right\| \leq K^{\prime} .
$$

Dividing by $m$ and multiplying with $i$ we obtain

$$
\left\|m \mathrm{i}\left[T_{f}^{(m)}, T_{g}^{(m)}\right]-T_{\mathrm{i}\left(C_{1}(f, g)-C_{1}(g, f)\right)}^{(m)}\right\|=O\left(\frac{1}{m}\right) .
$$

Using the asymptotics given by Theorem 2.3 (b) for the commutator we get

$$
\left\|T_{\{f, g\}-\mathrm{i}\left(C_{1}(f, g)-C_{1}(g, f)\right)}^{(m)}\right\|=O\left(\frac{1}{m}\right) .
$$


Taking the limit for $m \rightarrow \infty$ and using Theorem 2.3 (a) we get

$$
\left\|\{f, g\}-\mathrm{i}\left(C_{1}(f, g)-C_{1}(g, f)\right)\right\|_{\infty}=0 .
$$

Hence $\{f, g\}=\mathrm{i}\left(C_{1}(f, g)-C_{1}(g, f)\right)$. This shows (7).

\subsection{The uniqueness}

It is proven by induction using the asymptotics (9). Let $C_{j}(f, g)$ and $\tilde{C}_{j}(f, g)$ be two such systems of bilinear maps fulfilling the required properties. Assume $C_{j}=\tilde{C}_{j}$ for $j \leq N-2$. If we subtract the corresponding expressions in (9) and use the fact that $T^{(m)}$ is linear we obtain

$$
\left\|\frac{1}{m^{N-1}} T_{\left(C_{N-1}(f, g)-\tilde{C}_{N-1}(f, g)\right)}^{(m)}\right\| \leq \frac{K}{m^{N}} .
$$

Hence,

$$
\lim _{m \rightarrow \infty}\left\|T_{\left(C_{N-1}(f, g)-\tilde{C}_{N-1}(f, g)\right)}^{(m)}\right\|=0 .
$$

With Theorem 2.3 (a) it follows $C_{N-1}(f, g)=\tilde{C}_{N-1}(f, g)$. The induction starts with $N=1$. But here $C_{0}(f, g)=\tilde{C}_{0}(f, g)=f \cdot g$ is required.

\subsection{The associativity}

The proof employs the associativity of the operators used to construct the starproduct and again Theorem 2.3 (a). The relation $f \star(g \star h)=(f \star g) \star h$ can be rewritten in relations for the maps $C_{j}$ :

$$
\sum_{l=0}^{k} C_{l}\left(f, C_{k-l}(g, h)\right)=\sum_{l=0}^{k} C_{l}\left(C_{k-l}(f, g), h\right) .
$$

From Theorem 2.3 (a) we know $f=g \longleftrightarrow \lim _{m \rightarrow \infty}|| T_{f}^{(m)}-T_{g}^{(m)} \|=0$. Hence it is enough to apply the Toeplitz operator $T^{(m)}$ to the relation (31) and study the asymptotics of $T_{\text {left-hand side }}-T_{\text {right-hand side }}$. This is done by induction over $k$. $k=0: C_{0}\left(f, C_{0}(g, h)\right)=C_{0}\left(C_{0}(f, g), h\right)$ is true because $C_{0}(f, g)=f \cdot g$.

Assume the claim to be true up to level $k-1$. The equation (9) for $0 \leq r \leq k$ multiplied by $m^{r}(N=r+1)$ yields

$$
T_{C_{r}(f, g)}^{(m)}=m^{r} T_{f}^{(m)} T_{g}^{(m)}-\sum_{s=0}^{r-1} m^{r-s} T_{C_{s}(f, g)}+O\left(\frac{1}{m}\right) .
$$

Here the symbol $O\left(\frac{1}{m}\right)$ is shorthand for the statement that the difference of the operators on the left and on the right is an operator whose norm behaves like $O\left(\frac{1}{m}\right)$ for $m \rightarrow \infty$. In particular we obtain for $l=0,1, \ldots, k$

$$
T_{C_{l}\left(f, C_{k-l}(g, h)\right)}^{(m)}=m^{l} T_{f}^{(m)} T_{C_{k-l}(g, h)}^{(m)}-\sum_{s=0}^{l-1} m^{l-s} T_{C_{s}\left(f, C_{k-l}(g, h)\right)}+O\left(\frac{1}{m}\right) .
$$


Summation over $l$ yields

$$
T_{\text {1.h.s. }}^{(m)}=\sum_{l=0}^{k} m^{l} T_{f}^{(m)} T_{C_{k-l}(g, h)}^{(m)}-\sum_{l=0}^{k} \sum_{s=0}^{l-1} m^{l-s} T_{C_{s}\left(f, C_{k-l}(g, h)\right)}^{(m)}+O\left(\frac{1}{m}\right) .
$$

The second sum can be rewritten as

$$
-\sum_{r=1}^{k} m^{r} \sum_{l=r}^{k} T_{C_{l-r}\left(f, C_{k-l}(g, h)\right)}^{(m)}=-\sum_{r=1}^{k} m^{r} T_{\sum_{s=0}^{k-r} C_{s}\left(f, C_{k-r-s}(g, h)\right)}^{(m)} .
$$

For such sums we know by induction that (31) is valid. The same is done for the right hand side. If we subtract $T_{\text {r.h.s. }}^{(m)}$ from $T_{\text {l.h.s. }}^{(m)}$, it remains

$$
\sum_{l=0}^{k} m^{l} T_{f}^{(m)} T_{C_{k-l}(g, h)}^{(m)}-\sum_{l=0}^{k} m^{l} T_{C_{k-l}(m)}^{(m) g)} T_{h}^{(m)}+O\left(\frac{1}{m}\right) .
$$

By splitting the first sum into $l=0$ and $l \geq 1$ and using for the $l=0$ term the asymptotic (32) we obtain

$$
\begin{aligned}
& m^{0}\left(T_{f}^{(m)} m^{k} T_{g}^{(m)} T_{h}^{(m)}-\sum_{s=0}^{k-1} m^{k-s} T_{f}^{(m)} T_{C_{s}(g, h)}^{(m)}+O\left(\frac{1}{m}\right)\right)+\sum_{l=1}^{k} m^{l} T_{f}^{(m)} T_{C_{k-l}(g, h)}^{(m)} \\
& =m^{k} T_{f}^{(m)}\left(T_{g}^{(m)} T_{h}^{(m)}\right)+O\left(\frac{1}{m}\right) .
\end{aligned}
$$

A corresponding expression follows for the second sum. As difference remains

$$
m^{k}\left(T_{f}^{(m)}\left(T_{g}^{(m)} T_{h}^{(m)}\right)-\left(T_{f}^{(m)} T_{g}^{(m)}\right) T_{h}^{(m)}\right)+O\left(\frac{1}{m}\right) .
$$

Now we ended up with operators which are clearly associative, The operator coming with the $m^{k}$ term vanishes. Hence associativity follows from Theorem 2.3 (a).

\section{Additional properties}

The introduced star-product has important properties.

\subsection{Unit}

The unit of the algebra $C^{\infty}(M)$, the constant function 1 , will also be the unit in the star-product. Such star-products are sometimes called to have the property "null on constants".

Proposition 5.1 For the above introduced star-product we have

$$
1 \star g=g \star 1=g
$$

Equivalently,

$$
C_{k}(1, g)=C_{k}(g, 1)=0, \quad \text { for } \quad k \geq 1
$$


Proof. For $f \equiv 1$ we have $T_{f} \equiv i d$, resp. $T_{f}^{(m)} \equiv i d$. Also $C_{0}(1, g)=g=C_{0}(g, 1)$. Further with (20)

$$
A_{1}=D_{\varphi} T_{f} T_{g}-D_{\varphi} T_{f g}=D_{\varphi} T_{g}-D_{\varphi} T_{g} .
$$

Hence the symbol of $A_{1}$ vanishes. But this implies $C_{1}(1, g)=0=C_{1}(g, 1)$. The claim follows by trivial induction from (20) .

\subsection{Parity}

A star-product is said to fulfill the parity condition if

$$
\overline{f \star g}=\bar{g} \star \bar{f} .
$$

Considering the formal parameter to be real $(\bar{v}=v)$ this is equivalent to

$$
\overline{C_{k}(f, g)}=C_{k}(\bar{g}, \bar{f}), \quad k \geq 0 .
$$

We will show

Proposition 5.2 The above introduced star-product fulfills parity.

\section{Lemma 5.3}

$$
T_{f}^{(m)^{*}}=T_{\bar{f}}^{(m)} .
$$

Proof. Take any $s, t \in \Gamma_{h o l}\left(M, L^{m}\right)$. For the scalar product we calculate $\left(\Pi^{(m)}\right.$ is the projector defined in (15))

$$
\left\langle s, T_{f}^{(m)} t\right\rangle=\left\langle s, \Pi^{(m)} f t\right\rangle=\langle s, f t\rangle=\left\langle\bar{f}_{s}, t\right\rangle=\left\langle T_{\bar{f}}^{(m)} s, t\right\rangle .
$$

Hence the claim.

Proof. (Proposition 5.2) Recall that the identification of the sections of $L^{m}$ with equivariant functions on the circle bundle $Q$ is an isomorphy. Hence the definition of adjoint operators agree. For the global Toeplitz operator we obtain $T_{f}^{*}=$ $\prod_{m=0}^{\infty} T_{\bar{f}}^{(m)}=T_{\bar{f}}$. The star-product

$$
\bar{g} \star \bar{f}=\sum_{j=0}^{\infty} v^{j} C_{j}(\bar{g}, \bar{f})
$$

is given via the asymptotic expansion of

$$
T_{\bar{g}}^{(m)} \cdot T_{\bar{f}}^{(m)}=T_{g}^{(m)^{*}} \cdot T_{f}^{(m)^{*}}=\left(T_{f}^{(m)} \cdot T_{g}^{(m)}\right)^{*} .
$$


For the asymptotic expansion of the last expression we have

$$
\left(T_{f}^{(m)} \cdot T_{g}^{(m)}\right)^{*} \sim \sum_{j=0}^{\infty}\left(\frac{1}{m}\right)^{j} T_{C_{j}(f, g)}^{(m) *}=\sum_{j=0}^{\infty}\left(\frac{1}{m}\right)^{j} T_{\overline{C_{j}(f, g)}}^{(m)} .
$$

But this is the complex conjugate of the asymptotic expansion which defines $f \star g$. This shows (43)

By the parity condition we have on $C^{\infty}(M)[[v]]$ an anti-involution given by pointwise complex conjugation on the functions, and by considering the formal parameter to be real $(\bar{v}=v)$.

\subsection{Locality and separation of variables}

Recall that a star-product is local if for all $f, g \in C^{\infty}(M)$ the support supp $C_{j}(f, g)$ is contained in supp $f \cap \operatorname{supp} g$ for all $j \in \mathbb{N}_{0}$. Using Peetre's theorem and the fact that the $C_{j}$ are bilinear this implies that for a local star-product the $C_{j}$ can be given by bidifferential operators.

One way to proof locality is by studying the symbol calculus of degree zero Toeplitz operators which commute with the $S^{1}$ action in more detail (see [17,9]). Another possibility (which gives a different perspective) is to use the fact that the projection operators $\Pi^{(m)}$ can be expressed with the help of Berezin-Rawnsley's coherent states and the fact that the coherent states are "localizing" for $m \rightarrow \infty$. The details will appear in [19].

From the locality it follows that the star-product can be restricted to open subsets and defines compatible star-products there. For such star-products Karabegov introduced the notion of star-products with separation of variables [18] (Bordemann and Waldmannn [7] called them star-products of Wick type). In our convention this reads as $f \star k=f \cdot k$ and $k \star g=k \cdot g$ for (locally defined) holomorphic functions $g$, antiholomorphic functions $f$ and arbitrary functions $k$. The above introduced star-product will be a star-product with separation of variables.

More precisely, we expect that the $C_{j}$ are bidifferential operators of degree $(j, j)$ with only holomorphic derivatives in the first entry and only antiholomorphic derivatives in the second entry. See Section 5.5 for examples.

\subsection{Trace}

Proposition 5.4. (Bordemann, Meinrenken, Schlichenmaier) Let $f \in C^{\infty}(M)$ and let $n=\operatorname{dim}_{\mathbb{C}} M$. Denote the trace on $\operatorname{End}\left(\Gamma_{h o l}\left(M, L^{m}\right)\right)$ by $\operatorname{Tr}^{(m)}$ then

$$
\operatorname{Tr}^{(m)}\left(T_{f}^{(m)}\right)=m^{n}\left(\frac{1}{\operatorname{vol}\left(\mathbb{P}^{n}(\mathbb{C})\right)} \int_{M} f \Omega+O\left(m^{-1}\right)\right) .
$$

This result can also be found in [6]. There it was given only with a hint of its proof. Because it is central for the following let me give the details. 
Proof. Let us start with a real valued $f$. Then the operator $T_{f}$ and the components $T_{f}^{(m)}$ are self adjoint (see (45)). Let $d(m)=\operatorname{dim} \mathscr{H}^{(m)}$ and let $\lambda_{1}^{(m)}, \lambda_{2}^{(m)}, \ldots, \lambda_{d(m)}^{(m)}$ be the eigenvalues of the restriction of $T_{f}$ on $\mathscr{H}^{(m)}$. In particular, these are also the eigenvalues of $T_{f}^{(m)}$ on $\Gamma_{h o l}\left(M, L^{m}\right)$. Following [9] $\left(n=\operatorname{dim}_{\mathbb{C}} M\right)$ let

$$
\mu_{m}=\frac{1}{m^{n}} \sum_{i=1}^{d(m)} \delta\left(\lambda-\lambda_{i}^{(m)}\right)
$$

be the discrete spectral measure. By Theorem 13.13 of [9] it converges weakly to the limit measure

$$
\mu(g)=\gamma_{M} \int_{M} g(f(z)) \Omega(z)
$$

with a universal constant $\gamma_{M}$ only depending on the manifold $M$. An important intermediate result there is the asymptotic expansion (Equation 13.13 in [9])

$$
\mu_{m}(g) \sim \sum_{r=-n}^{\infty} a_{r}(g) m^{r+n} .
$$

For $g \equiv 1$ we obtain

$$
\frac{1}{m^{n}} \sum_{i=1}^{d(m)} \lambda_{i}^{(m)}=\frac{1}{m^{n}} \operatorname{Tr}^{(m)} T_{f}^{(m)}=\gamma_{M} \int_{M} f \Omega+O\left(\frac{1}{m}\right) .
$$

To calculate $\gamma_{M}$ we evaluate (54) for $f \equiv 1$ (i.e., $T_{f}^{(m)}=i d$ ) and obtain

$$
\gamma_{M}=\frac{\operatorname{dim} \Gamma_{h o l}\left(M, L^{m}\right)}{m^{n} \cdot \operatorname{vol}(M)}+O\left(\frac{1}{m}\right) .
$$

Note that (see p.113 and Thm 5.22 in [25])

$$
\operatorname{dim} \Gamma_{h o l}\left(M, L^{m}\right)=\frac{\operatorname{vol}(M)}{\operatorname{vol}\left(\mathbb{P}^{n}(\mathbb{C})\right)} \cdot m^{n}+O\left(m^{n-1}\right) .
$$

Hence $\gamma_{M}=\operatorname{vol}\left(\mathbb{P}^{n}(\mathbb{C})\right)^{-1}$. In particular the coefficient depends only on the dimension of $M$. This shows the claim for real valued $f$. For complex valued $f$ it follows from linearity by considering real and imaginary parts separately. In [25] for $M$ the restriction $\omega_{F S \mid M}$ of the Fubini-Study Kähler form was used to define the volume. Here we have to work with the form $\omega$. Because the de Rham classes of both forms coincide and the Kähler forms are closed, the volume will be the same.

From (53) follows the asymptotic expansion for $m \rightarrow \infty$ (see also [8])

$$
\operatorname{Tr}^{(m)}\left(T_{f}^{(m)}\right) \sim m^{n}\left(\sum_{j=0}^{\infty}\left(\frac{1}{m}\right)^{j} \tau_{j}(f)\right), \quad \text { with } \tau_{j}(f) \in \mathbb{C} .
$$


We define the $\mathbb{C}[[v]]$-linear map

$$
\operatorname{Tr}: C^{\infty}(M)[[v]] \rightarrow v^{-n} \mathbb{C}[[v]], \quad \operatorname{Tr} f:=v^{-n} \sum_{j=0}^{\infty} v^{j} \tau_{j}(f),
$$

such that for $f \in C^{\infty}(M)$ the $\tau_{j}(f)$ are given by the asymptotic expansion (57) and for arbitrary elements by $\mathbb{C}[[v]]$-linear extension.

Proposition 5.5 The map $\operatorname{Tr}$ is a trace, i.e., we have

$$
\operatorname{Tr}(f \star g)=\operatorname{Tr}(g \star f) .
$$

Proof. By $\mathbb{C}[[v]]$-linearity it is enough to show this for $f, g \in C^{\infty}(M)$. The element $f \star g-g \star f$ is given by the asymptotic expansion of $T_{f}^{(m)} \cdot T_{g}^{(m)}-T_{g}^{(m)} \cdot T_{f}^{(m)}$. Hence $\operatorname{Tr}(f \star g-g \star f)$ is given by the expansion of

$$
\operatorname{Tr}^{(m)}\left(T_{f}^{(m)} \cdot T_{g}^{(m)}-T_{g}^{(m)} \cdot T_{f}^{(m)}\right) .
$$

But for every $m$ this vanishes. Hence (59) follows.

\subsection{Examples}

For the sphere $S^{2}$, resp. $\mathbb{P}^{1}(\mathbb{C})$ with Kähler form

$$
\omega=\frac{\mathrm{i}}{(1+z \bar{z})^{2}} d z \wedge d \bar{z}
$$

and the hyperplane bundle as quantum line-bundle explicit calculations ${ }^{2}$ of the author (not published) yield (using $T^{(m)}(f):=T_{f}^{(m)}$ )

$$
\lim _{m \rightarrow \infty}\left\|m\left(T^{(m)}(f) T^{(m)}(g)-T^{(m)}(f g)\right)+T^{(m)}\left((1+z \bar{z})^{2} \frac{\partial f}{\partial z} \frac{\partial g}{\partial \bar{z}}\right)\right\|=0 .
$$

This implies

$$
C_{1}(f, g)=-(1+z \bar{z})^{2} \frac{\partial f}{\partial z} \frac{\partial g}{\partial \bar{z}} .
$$

For the case of Riemann surfaces of genus $g \geq 2$ more than half of the article [20] by Klimek and Lesniewski deals with the proof of the fact corresponding to (62). In the realization of the Riemann surface $M$ as quotient space $\{z \in \mathbb{C}|| z \mid<1\} / G$ with $G$ a Fuchsian subgroup of $\mathrm{SU}(1,1)$ acting by fractional linear transformations one takes as Kähler form the $\mathrm{SU}(1,1)$ invariant form

$$
\omega=\frac{2 \mathrm{i}}{(1-z \bar{z})^{2}} d z \wedge d \bar{z}
$$

\footnotetext{
${ }^{2}$ Not following the lines of the proof in Section 4 but working with a basis of the sections of the bundles.
} 
The corresponding quantum line bundle is the canonical line bundle, i.e., the bundle whose local sections are the holomorphic differentials. From their results follows

$$
C_{1}(f, g)=-\frac{1}{2}(1-z \bar{z})^{2} \frac{\partial f}{\partial z} \frac{\partial g}{\partial \bar{z}} .
$$

\subsection{Deformation quantization via geometric quantization}

Via Tuynman's relation (14) the operator $Q^{(m)}$ of geometric quantization corresponding to the function $f$ can be expressed by the Toeplitz operator $T^{(m)}$ corresponding to the function $f-\frac{1}{2 m} \Delta f$. Theorem 2.2 shows that the asymptotic expansion

$$
Q_{f}^{(m)} \cdot Q_{g}^{(m)} \sim \sum_{j=0}^{\infty}\left(\frac{1}{m}\right)^{j} Q_{D_{j}(f, g)}^{(m)},
$$

with suitable $D_{j}(f, g) \in C^{\infty}(M)$ is well-defined in the precise sense as expressed in the theorem there. We set

$$
f \star_{G} g:=\sum_{j=0}^{\infty} v^{j} D_{j}(f, g) .
$$

The first two terms calculate as

$$
\begin{gathered}
D_{0}(f, g)=f \cdot g \\
D_{1}(f, g)=C_{1}(f, g)+\frac{1}{2}(\Delta(f \cdot g)-\Delta f \cdot g-f \cdot \Delta g),
\end{gathered}
$$

where the $C_{j}$ are the coefficients of the Berezin-Toeplitz star-product. In particular the conditions (7) are fulfilled for the $D$ 's. Hence, this defines indeed a star-product.

In fact more is valid. If we introduce the linear maps

$$
B^{(m)}(f):=f-\frac{1}{2 m} \Delta f
$$

and the $\mathbb{C}[[v]]$-linear map induced by

$$
B(f):=f-v \frac{\Delta}{2} f=\left(i d-v \frac{\Delta}{2}\right) f
$$

on $C^{\infty}(M)[[v]]$ we can rewrite (66)

$$
T_{B^{(m)}(f)}^{(m)} \cdot T_{B^{(m)}(g)}^{(m)} \sim \sum_{j=0}^{\infty}\left(\frac{1}{m}\right)^{j} T_{B^{(m)}\left(D_{j}(f, g)\right)}^{(m)} .
$$

Taking the asymptotics we get $B(f) \star B(g)=B\left(f \star_{G} g\right)$. Note that $B(f) \bmod v=$ $f, B(1)=1$, and that $B$ is invertible. The inverse is given by

$$
B^{-1}=i d+\sum_{k=1}^{\infty} \frac{1}{2^{k}} v^{k} \Delta^{k}
$$


Recall that two star-products (over the same manifold) are equivalent if there exists a $\mathbb{C}[[v]]$-algebra isomorphism inducing the identity on the zero order part. This implies

Proposition 5.6 The star-product of geometric quantization is equivalent to the star-product of Berezin-Toeplitz quantization.

Acknowledgements. As the basics of the presented work goes back to joint work with M. Bordemann and E. Meinrenken it is a pleasure for me to thank them for all their inspirations. I also like to thank the Erwin Schrödinger International Institute for Mathematical Physics in Vienna for its hospitality, and F. Haslinger, P. Michor and H. Upmeier for the invitation to participate in one of the activities at the institute. During the stay there the main part of this article was written up.

\section{References}

1. Bayen, F., Flato, M., Fronsdal, C., Lichnerowicz, A., and Sternheimer, D.: Quantum mechanics as a deformation of classical mechanics, Lett. Math. Phys. 1 (1975/77), 521-530; Deformation theory and quantization, Part I and II, Ann. Physics 111 (1978), 61-110, 111-151.

2. Berceanu, St. and Schlichenmaier, M.: Coherent state embeddings, polar divisors and Cauchy formulas,preprint math.QA/9902066, to appear in J. Geom. Phys.

3. Berezin, F.A.: Quantization, Math. USSR-Izv. 8 (1974), 1109-1165; Quantization in complex symmetric spaces, Math. USSR-Izv. 9 (1975), 341-379.

4. Bertelson, M., Cahen, M., and Gutt, S.: Equivalence of star products, Classical Quantum Gravity 14 (1997), A93-A107.

5. Bordemann, M., Hoppe, J., Schaller, P., and Schlichenmaier, M.: $g l(\infty)$ and geometric quantization, Comm. Math. Phys. 138 (1991), 209-244.

6. Bordemann, M., Meinrenken, E., and Schlichenmaier, M.: Toeplitz quantization of Kähler manifolds and $g l(n), n \rightarrow \infty$ limits, Comm. Math. Phys. 165 (1994), 281-296.

7. Bordemann, M. and Waldmann, St.: A Fedosov star product of the Wick type for Kähler manifolds, Lett. Math. Phys. 41 (1997), 243-253.

8. Borthwick, D., Paul, D., and Uribe, A.: Semiclassical spectral estimates for Toeplitz operators, Ann. Inst. Fourier, Grenoble 48 (1998), 1189-1229.

9. Boutet de Monvel, L. and Guillemin, V.: The spectral theory of Toeplitz operators, Ann. Math. Studies, Nr.99, Princeton University Press, Princeton, 1981.

10. Cahen, M., Gutt, S., and Rawnsley, J.: Quantization of Kähler manifolds II, Trans. Amer. Math. Soc. 337 (1993), 73-98.

11. De Wilde, M. and Lecomte, P.: Existence of star products and of formal deformations of the Poisson-Lie algebra of arbitrary symplectic manifolds, Lett. Math. Phys. 7 (1983), 487-496.

12. Deligne, P.: Letter to Bordemann, Meinrenken and Schlichenmaier (March 1994). Answer by Meinrenken, 1994.

13. Deligne, P.: Déformation de l'algèbre des fonctions d'une variété symplectique: Comparaison entre Fedosov et De Wilde, Lecomte, Selecta Math. (N.S.) 1 (1995), 667-697.

14. Fedosov, B.V.: Deformation quantization and index theory, Akademie Verlag, Berlin, 1996.

15. Fedosov, B.V.: Deformation quantization and asymptotic operator representation, Funktional Anal. i. Prilozhen. 25 (1990), 184-194; A simple geometric construction of deformation quantization, J. Diff. Geom. 40 (1994), 213-238.

16. Guillemin, V.: Some classical theorems in spectral theory revisited, in: L. Hörmander, (ed.), Seminars on singularities of solutions of linear partial differential equations, Ann. of Math. Stud., 91, Princeton University Press, Princeton, NJ, 1979, pp. 219-259. 
17. Guillemin, V.: Star products on pre-quantizable symplectic manifolds, Lett. Math. Phys. 35 (1995), 85-89.

18. Karabegov, A.: Deformation quantization with separation of variables on a Kähler manifold, Comm. Math. Phys. 180 (1996), 745-755.

19. Karabegov, A. and Schlichenmaier, M.: in preparation.

20. Klimek, S. and Lesniewski, A., Quantum Riemann surfaces: II. The discrete series, Lett. Math. Phys. 24 (1992), 125-139.

21. Kontsevich, M.: Deformation quantization of Poisson manifolds I, preprint q-alg/9709040.

22. Landsman, N.P.: Mathematical topics between classical and quantum mechanics, Springer, Berlin, Heidelberg, New York, 1998.

23. Moreno, C.: *-products on some Kähler manifolds, Lett. Math. Phys. 11 (1986), 361-372.

24. Moreno, C. and Ortega-Navarro, P.: *-products on $D^{1}(\mathbb{C}), S^{2}$ and related spectral analysis, Lett. Math. Phys. 7 (1983), 181-193.

25. Mumford, D.: Complex projective varieties, Springer, Berlin, Heidelberg, New York, 1976.

26. Nest, R. and Tsygan, B.: Algebraic index theory, Comm. Math. Phys. 172 (1995), 223-262; Algebraic index theory for families, Adv. Math. 113 (1995), 151-205.

27. Omori, H., Maeda, Y., and Yoshioka, A.: Weyl manifolds and deformation quantization, $A d v$. Math. 85 (1991), 224-255; Existence of closed star-products, Lett. Math. Phys. 26 (1992), 284-294.

28. Reshetikhin, N. and Takhtajan, L.:Deformation quantization of Kähler manifolds, preprint math/9907171.

29. Rieffel, M.A.: Deformation quantization and operator algebras, in: W. Arveson and R. Douglas, (eds.), Operator theory: Operator algebras and applications, Proc. Sympos. Pure Math., 51, Amer. Math. Soc., Providence, RI, 1990, pp. 411-423.

30. Rieffel, M.A.: Questions on quantization, in: Liming Ge et al., (eds.), Operator algebras and operator theory (Shanghai 1997), Contemp. Math., 228, Amer. Math. Soc., Providence, RI, 1998, pp. 315-326.

31. Schlichenmaier, M.: Berezin-Toeplitz quantization of compact Kähler manifolds, in: A. Strasburger et al. (eds.), Quantization, Coherent States and Poisson Structures, Proc. XIV'th Workshop on Geometric Methods in Physics (Białowieza 1995), Polish Scientific Publisher, Warsaw, 1998, pp. 101-115 ( q-alg/9601016).

32. Schlichenmaier, M.: Zwei Anwendungen algebraisch-geometrischer Methoden in der theoretischen Physik: Berezin-Toeplitz-Quantisierung und globale Algebren der zweidimensionalen konformen Feldtheorie, Mannheim, 1996.

33. Schlichenmaier, M.: Deformation quantization of compact Kähler manifolds via BerezinToeplitz operators, in: H.-D. Doebner, P. Nattermann, and W. Scherer, (eds.), Proceedings of the XXI International Colloquium on Group Theoretical Methods in Physics (Goslar 1996), World Scientific, Singapore, 1997, pp. 396-400.

34. Schlichenmaier, M.: Berezin-Toeplitz quantization and Berezin symbols for arbitrary compact Kähler manifolds, preprint math.QA/9902066, to appear in Rep. on Math. Phys.

35. Tuynman, G.M.: Quantization: Towards a comparison between methods, J. Math. Phys. 28 (1987), 2829-2840.

36. Weinstein, A. and $\mathrm{Xu}, \mathrm{P} .:$ Hochschild cohomology and characteristic classes for star-products, in: A. Khovanskij et al., (eds.), Geometry and differential equations, Amer. Math. Soc. Transl. Ser. 2, 186, Amer. Math. Soc., Providence, RI, 1998, pp. 177-194. 